\title{
Discussion on the Integration and Optimization Plan of Natural Reserve-Take Gannan Tibetan Autonomous Prefecture as an example
}

\author{
Boqiang Zhai ${ }^{1, a^{*}}$, Xitun Yuan ${ }^{2, b}$ \\ ${ }^{1}$ Xi'an University of Science and Technology, Xi'an, Shaanxi, China \\ ${ }^{2} \mathrm{Xi}$ 'an University of Science and Technology, Xi'an, Shaanxi, China
}

\begin{abstract}
The integration and optimization of nature reserves is an important part of the new round of land and space planning, and it is also an important part of building a system of nature reserves with national parks as the main body. This article takes Gannan Tibetan Autonomous Prefecture, which has many nature reserves and relatively complex conditions as an example, to summarize and study the technical and operational issues involved in the integration and optimization of 30 different types of nature reserves, natural parks and scenic spots in the region. We propose an integration and optimization plan that fits the region, focusing on the treatment of the overlapping and distributed residential land, basic farmland, and major construction projects of each protected area, and provide reasonable suggestions for the integration and optimization of the construction of natural reserves with Chinese characteristics.
\end{abstract}

\section{Preface}

According to the concept given by the International Union for Conservation of Nature (IUCN), a protected area is a clearly defined geographical area that is recognized by law or other effective means and aims to achieve long-term preservation of nature and the services and cultural values of related ecosystems. Area [1]. In our country, nature reserves are defined or confirmed by governments at all levels in accordance with the law, and long-term protection of important natural ecosystems, natural relics, natural landscapes and the natural resources, ecological functions and cultural values they carry is carried out on land or sea areas. [2]. Internationally, there are corresponding regulations and specific consensus on nature reserves. Since China's nature reserves have not undergone systematic overall planning, they are mostly established by department-led and local declarations from the bottom up. They are limited to the reality of local division and division of departments. Therefore, there are a series of problems, such as imperfect top-level design and laws and regulations. Unsound, unreasonable spatial layout, unscientific classification system, unsmooth management system, unclear property rights responsibilities, mainly manifested in fuzzy positioning, multiple management, overlapping, unclear boundaries, unreasonable zoning, and prominent contradictions between protection and development, etc. , The phenomenon of spatial division and fragmentation of the ecosystem has occurred; an overall efficient, organically connected, and complementary nature reserve system has not yet been formed, and the management efficiency has been reduced, hindering the overall function; it cannot truly provide the public with high-quality ecological products and support the economy The sustainable development of society is incompatible with the development requirements of the new era. Under the new situation, the reconstruction of the natural reserve system is urgent. [3]

Establishing a protected area system with national parks as the main body is a major measure to implement Xi Jinping's thoughts on ecological civilization and a major reform task proposed by the 19th National Congress of the Communist Party of China. The concept of ecological civilization that respects nature, conforms to nature, and protects nature must be firmly established. The integration and optimization of nature reserves is an important basic work for establishing a system of nature reserves with national parks as the main body. Through integration and optimization, scientifically solve the overlapping, unclear boundaries, multiple management, unclear rights and responsibilities, protection and development of nature reserves, etc. Real conflicts and problems left over from history: At the same time, other ecological spaces with conservation value should be included in the corresponding nature reserves, and the ecological space should be effectively expanded, so as to ensure that all necessary protections are maintained.

Lay the foundation for the establishment of a natural protected area system with scientific classification, reasonable layout, strong protection and effective management. [4]

*Corresponding author: a904256266@qq.com 


\section{Overview of the study area}

Gannan Prefecture is one of the ten Tibetan autonomous prefectures in the country. It is located in the southwestern part of Gansu Province, in the transitional zone between the northeastern edge of the Qinghai-Tibet Plateau and the western part of the Loess Plateau. It is connected to Aba Prefecture in Sichuan to the south, Huangnan Prefecture and Guoluo Prefecture in Qinghai to the southwest, and to the east and north. It is adjacent to Longnan City, Dingxi City and Linxia Prefecture. The geographical coordinates are between $100^{\circ} 46^{\prime} \sim 104^{\circ}$ $44^{\prime}$ east longitude and $33^{\circ} 06^{\prime} \sim 36^{\circ} 10^{\prime}$ north latitude. The total area of the whole prefecture is 3.66 million hectares. The altitude is between 1073 and 4920 meters, and the average temperature is $1.7^{\circ} \mathrm{C}$. The administrative region involves 98 towns and villages in 7 counties and 1 city. Up to now, the prefecture has established 30 nature reserves of various levels and types, with a total area of 1.5 million hectares, accounting for $41.0 \%$ of the total land area of the state, of which 15 organized towns, 101 administrative villages, 415 natural villages, 23,040 households, 104,204 people.

Table 1 Status of natural protected areas before integration and optimization in Gannan Prefecture

\begin{tabular}{|c|c|c|c|}
\hline Nature Reserve Name & Level & Area & Management agency \\
\hline Hezuo Provincial Forest Park & Provincial & 590.69 & Cooperative City Government \\
\hline Xiahe Ganjia Baishiya Provincial Geopark & Provincial & 5734.2 & Xiahe County Government \\
\hline Yeliguan National Forest Park & National level & 79574.81 & Taohe Ecological Construction Bureau \\
\hline Yeliguan National Geopark & National level & 13840.81 & Lintan County Government \\
\hline Yeliguan Scenic Area & Provincial & 30439.21 & Lintan County Government \\
\hline Yangsha Provincial Forest Nature Park & Provincial & 5396.03 & Taohe Ecological Construction Bureau \\
\hline Taohe National Nature Reserve, Gansu & National level & 287613.9 & Gansu Forestry and Grass Bureau \\
\hline Gansu Dayu National Forest Park & National level & 27942.01 & Taohe Nature Reserve Administration \\
\hline Dayugou Geopark, Zhuoni County & Provincial & 55416.43 & Taohe Nature Reserve Administration \\
\hline Kache Provincial Forest Park & Provincial & 14419.37 & Taohe Nature Reserve Administration \\
\hline Cheba Provincial Forest Park & Provincial & 13257.64 & Taohe Nature Reserve Administration \\
\hline Xiabagou Provincial Forest Park & Provincial & 4596.59 & Taohe Nature Reserve Administration \\
\hline Jiudongshan Provincial Forest Park, Zhuoni County & Provincial & 748.39 & Zhuoni County Government \\
\hline Gansu Yellow River Shouqu National Nature Reserve & National level & 203057.89 & Gansu Forestry and Grass Bureau \\
\hline $\begin{array}{l}\text { Natural Reserve of Indigenous Fishes in Qinghai-Tibet Plateau, } \\
\text { Maqu, Gansu Province }\end{array}$ & Provincial & 27415.26 & Maqu County Government \\
\hline Gansu Duoer National Nature Reserve & National level & 54401.24 & Gansu Forestry and Grass Bureau \\
\hline Bailongjiang Axia Provincial Nature Reserve, Gansu & Provincial & 130495.88 & Bailong River Forest Management Bureau \\
\hline Zhagana National Geopark, Diebu, Gannan Prefecture & National level & 18357.57 & Diebu County Government \\
\hline Lazikou National Forest Park & National level & 48427.97 & Diebu Ecological Construction Bureau \\
\hline Zhagana Provincial Forest Park, Diebu, Gansu & Provincial & 35896.14 & Diebu County Government \\
\hline Gansu Bailongjiang Chagangliang Provincial Nature Reserve & Provincial & 82915.44 & Bailong River Forest Management Bureau \\
\hline Bailongjiang Boyuhe Provincial Nature Reserve of Gansu & Provincial & 22005.09 & Bailong River Forest Management Bureau \\
\hline Daxiagou National Forest Park & National level & 4110.63 & Zhouqu County Government \\
\hline Shatan Forest Park & Provincial & 17488.44 & Zhouqu Ecological Construction Bureau \\
\hline Laga Mountain Provincial Forest Park & Provincial & 1355.5 & Zhouqu County Government \\
\hline Boyu Provincial Forest Park & Provincial & 13258.81 & Baishui River Ecological Construction Bureau \\
\hline Gansu Gahai-Zecha National Nature Reserve & National level & 247678.63 & Gansu Forestry and Grass Bureau \\
\hline Provincial Zecha Stone Forest Geopark, Luqu County, Gansu Province & Provincial & 24963.26 & Luqu County Government \\
\hline Zecha Provincial Forest Park & Provincial & 24963.26 & Luqu County Government \\
\hline Langmusi Provincial Scenic Are & Provincial & 3208.41 & Luqu County Government \\
\hline
\end{tabular}

\section{Problems with protected area}

\subsection{Serious spatial overlap}

There are serious overlapping problems among the 30 nature reserves in the state, with a total overlapping area of 237,800 hectares.

The Zecha Stone Forest Geological Park, Zecha Provincial Forest Park, Langmusi Scenic Area and Gahai Zecha National Nature Reserve in Luqu County, Gansu Province overlap 24963.26 hectares, 24963.26 hectares, and 3208.41 hectares respectively.

Dayu National Forest Park, Zhuoni County Dayugou Geological Park, Truck Provincial Forest Park, Cheba Provincial Forest Park, Xiabagou Provincial Forest Park and Gansu Taohe National Nature Reserve overlap 27942.01 hectares and 55416.43 hectares respectively, 14419.37 hectares, 13257.64 hectares, 4596.59 hectares

Yeliguan National Forest Park: Lintan County Yeliguan National Geopark and Yeliguan National Forest Park overlap 13,840.81 hectares, Yeliguan Scenic Area and Lintan County Yeliguan National Geopark

The overlap is 13,836.84 hectares, and the Yeliguan National Forest Park, the scenic area of Yeliguan, overlaps 18312.62 hectares.

Gansu Yellow River Shouqu National Nature Reserve: Maqu Qinghai-Tibet Plateau Native Fish Nature Reserve has an overlapping area of 368.41 hectares.

\subsection{There are a large number of residential areas and basic farmland in the protected area}

Among the 30 nature reserves in the whole state, 14 nature reserves have basic farmland, and the permanent basic farmland area is 19194.02 hectares. The core area of the nature reserve is 78.78 hectares of permanent basic farmland, the buffer zone is 7,694hectares, and the experimental area is 11420.29 hectares of permanent basic farmland. The permanent basic farmland in the natural park is 4600.7 hectares.

There are 15 organic towns, 101 administrative villages, 23,040 households, and 104,204 people in the 30 nature reserves of the whole prefecture. Among them, there are 1396 households with 4,431 persons in the core area of the nature reserve, 6 administrative villages in the buffer zone of the nature reserve, 1960 households with 6468 persons, the experimental area of the nature reserve has 15 townships, 95 administrative villages, 19,694 households with 93,305 persons. 


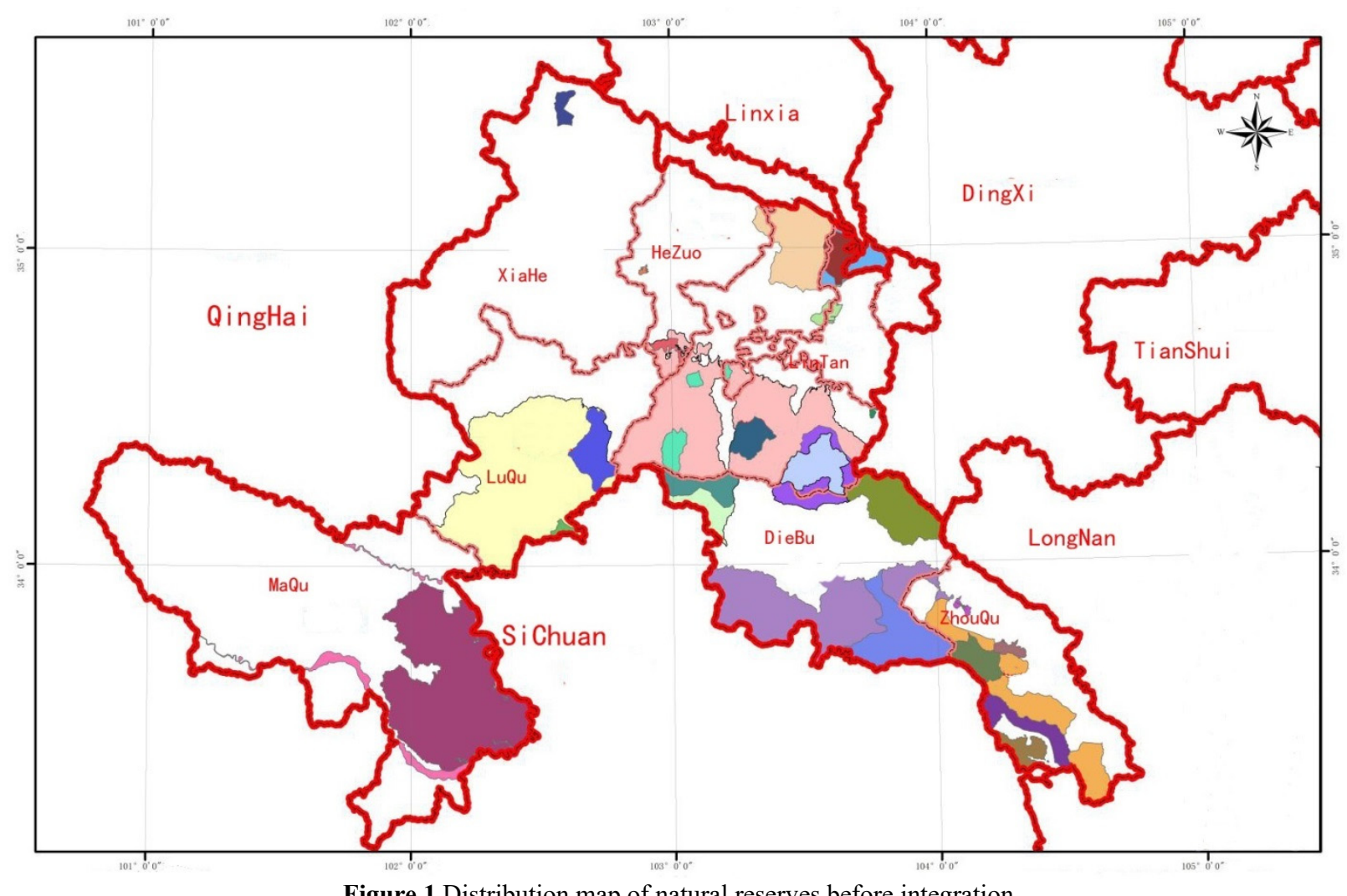

Figure 1 Distribution map of natural reserves before integration

\subsection{Existence of mining rights and major construction projects in the protected area}

There are 68 cases involving mining rights in 8 nature reserves in Gannan Prefecture, of which 37 cases are prospecting. There were 4 mining cases and 27 sand quarrying cases.

There are a total of 25 major transportation construction projects at the national, provincial and state levels in the nature reserves in Gannan Prefecture. G1816 Wuhai-Maqin National Expressway cooperation to Saillong (Ganqing boundary) section project, passing through the original experimental area 14.1 kilometers; Xining-Chengdu Railway (Gannan) section, passing through the Gahaizecha National Nature Reserve experimental area $49.9 \mathrm{~km}$. Line G248 Jiangguo River to Diebu Class II Highway (Zagul Town to Diebu Section), passing through the original core area and the original buffer zone for 20 kilometers; S581 Muer Town to Kaba Class II Highway (Dayugou to Kaba Section), Crossing the core reserve (former buffer zone) 9.0 kilometers; S20 Lianghekou-Langmusi Expressway (Lianghekou to Diebu Yiwa section), crossing the experimental area 6.18 kilometers; S209 Gonghe-Jiuzhaigou highway (Ma Ya to Jiuzhaigou section) ), passing through the original core area $6.72 \mathrm{~km}$, the original buffer zone $4.8 \mathrm{~km}$, the original experimental area $40.11 \mathrm{~km}$, and the core protection area $11.52 \mathrm{~km}$

\section{Integration and optimization measures}

According to the "Guiding Opinions on Establishing a Natural Reserve System with National Parks as the Main
Body" issued by the General Office of the Central Committee of the Communist Party of China and the General Office of the State Council, this integration and optimization follows the following principles:

(1)Scientific evaluation and reasonable adjustment. On the basis of biodiversity assessment, a scientific evaluation system will be established, focusing on resolving actual conflicts and historical issues. Make preparations for determining a reasonable optimization and adjustment plan.

(2)It should be fully delineated and guaranteed. Maintain the integrity of the natural ecosystem and the connectivity of ecological corridors, combine entry and transfer, protect all places that should be protected, and plan to establish a number of new nature reserves and other nature reserves.

(3)Seek truth from facts, simple and easy. The area of nature reserves is not preset, functional zoning is simplified, differentiated management and control measures are adopted, and transition periods are set for the gradual withdrawal of ecological relocation, permanent basic farmland, towns and villages, and mining rights, and strengthen the protection of property rights in accordance with the law and advance steadily.

(4)Make overall coordination and do a good job of connection. Combining the preparation of territorial and spatial planning, linking up the three control line regulations, launching simultaneously with the ecological protection red line assessment notice, and coordinating with the verification and rectification of permanent basic farmland, and rationally arranging production, living, and ecological spaces [4].

Mainly did the following work: 


\subsection{Revocation of completely overlapping nature reserves}

In the overlapping areas of nature reserves, comprehensive consideration is given to the protection level, protection objects, protection intensity, historical evolution, management level, etc., and only one protection area is retained through integration to achieve "a set of institutions, a name, and a brand". According to the protection level, the protection intensity is from high to low. As the level of nature reserves is higher than that of various natural parks, 10 nature parks are planned to be cancelled during the integration and optimization process. Table2:

Table 2 Gannan Prefecture intends to revoke nature reserves

\begin{tabular}{|c|c|c|c|}
\hline Nature Reserve Name & Level & Area & Reason for cancellation \\
\hline Yeliguan Scenic Area & Provincial & 30439.21 & Overlap with other high-level nature reserves \\
\hline Gansu Dayu National Forest Park & National level & 27942.01 & Overlap with other high-level nature reserves \\
\hline Dayugou Geopark, Zhuoni County & Provincial & 55416.43 & Overlap with other high-level nature reserves \\
\hline Kache Provincial Forest Park & Provincial & 14419.37 & Overlap with other high-level nature reserves \\
\hline Cheba Provincial Forest Park & Provincial & 13257.64 & Overlap with other high-level nature reserves \\
\hline Xiabagou Provincial Forest Park & Provincial & 4596.59 & Overlap with other high-level nature reserves \\
\hline Shatan Forest Park & Provincial & 17488.44 & Overlap with other high-level nature reserves \\
\hline Gansu Gahai-Zecha National Nature Reserve & National level & 247678.63 & Overlap with other high-level nature reserves \\
\hline Zecha Provincial Forest Park & Provincial & 24963.26 & Overlap with other high-level nature reserves \\
\hline Langmusi Provincial Scenic Area & Provincial & 3208.41 & Overlap with other high-level nature reserves \\
\hline
\end{tabular}

\subsection{Merge and transform into nature reserves or other nature reserves on the edge}

For nature reserves that cross adjacent parts, the principle of low-level merging and high-level conversion is adopted. The areas involved in this integration and optimization process include, see the attached table 3 for details

Table 3 Consolidation and transformation of natural reserves in Gannan Prefecture

\begin{tabular}{|l|l|l|l|}
\hline \multicolumn{1}{|c|}{ Nature Reserve Name } & Level & \multicolumn{1}{|c|}{ Overlapping protected area names } \\
\hline $\begin{array}{l}\text { Gansu Yellow River Shouqu National Nature } \\
\text { Reserve }\end{array}$ & National level & $\begin{array}{l}\text { Natural Reserve of Indigenous Fishes in Qinghai-Tibet Plateau, Maqu, } \\
\text { Gansu Province }\end{array}$ & 369.83 \\
\hline Gansu Duoer National Nature Reserve & National level & Bailongjiang Axia Provincial Nature Reserve, Gansu \\
\hline
\end{tabular}

\subsection{Transferring out residential land and basic farmland at the edge of centralized contiguous protected areas}

Due to historical reasons and Gannan Prefecture belongs to ethnic minority gathering areas, most areas belong to poverty-stricken areas. When the scope of the protection area was originally delineated, some residential areas and basic farmland were included in the protection area. After years of development, the protection and The problem of development is very prominent. On the one hand, people's livelihood and production are strongly dependent on protected areas, and on the other hand, the protection of protected areas cannot be handled well. This integration and optimization takes into account the issues of protection and development. The concentrated and contiguous residential land and basic farmland around the protected area are transferred out of the protected area. After many discussions, the area of the protected area is larger in some areas, and It may be in a situation where the sunroof is properly opened.

\subsection{Reserve channels for major construction projects}

In recent years, major construction projects in Gannan Prefecture have been carried out in an orderly manner. Among them, many national key projects, especially transportation projects, will pass through general control areas of nature reserves, and some also involve passing through core protection areas. For some key projects, those in the protected area will be ensured through changes in planning and reserved passages.Protect nature reserves while developing the project.

\subsection{Rectify illegal water conservancy mining in protected areas and illegal points detected in the Green Shield operation}

The central environmental protection inspector and the "Green Shield" special action on the rectification of ecological and environmental problems. During the central environmental protection inspection, no ecological and environmental problems related to Gannan Prefecture's nature reserve were found. In 2017 and 2018, the "Green Shield Operation" cleaned up and identified 147 ecological and environmental problems. So far, 147 items have passed county-level acceptance; 134 items have passed state-level review and recognition; 13 items have been rectified and awaited acceptance at the provincial and state levels. Natural parks have no ecological environment violations of laws and regulations.

\subsection{Transfer to areas with higher protection value around the protected area and add grassland parks}

Gannan Prefecture is rich in grassland forestry resources. This integration and optimization has been transferred to some areas with high conservation value and two grassland parks have been added. Gansu Gahaizecha 
National Nature Reserve was transferred to the source area of the Tao River in Liqiaru, Luqu County, 6452.93 hectares. The transferred area is an important water conservation area for the Tao River, a tributary of the Yellow River. Forests, wetlands and grassland resources are alternately distributed and biodiversity Rich in sex, high protection value. Gansu Yellow River Shouqu National Nature Reserve transferred into five rivers, Yaodarqu, Niangyiqu, Cheluoqu, Naaiqu, and Tangqu, covering an area of 717.20 hectares. The five rivers in the area are an important part of the Manrima, Zerima, Qihama marsh wetlands and river wetlands. The reserve is an important water conservation area and reservoir in the upper reaches of the Yellow River, and is the main water supply for the main stream of the Yellow River. One of the districts.

In order to fully promote the integrated development of forest and grass, and to protect and restore grasslands, the National Forestry and Grass Administration decided to carry out pilot projects to promote the construction of national natural grassland parks. Gannan Prefecture has added two new grassland parks during this integration and optimization, namely Awancang National Grassland Natural Park in Gansu Province in Maqu County and Gansu Meiren National Grassland Natural Park in Hezuo City

\section{Integration and optimization plans and suggestions}

\subsection{Integrated optimization plan}

According to the integration and optimization measures, 22 nature reserves remain after the integration and optimization. There is no overlap between the protected areas; the basic farmland and residential areas in the protected areas that can be transferred have been adjusted accordingly.After evaluation and field survey, the area was transferred to a natural reserve, the scope of the protected area was enlarged, and two national grassland parks were added.

Table 4 Status of natural protected areas after integration and optimization in Gannan Prefecture

\begin{tabular}{|c|c|c|c|}
\hline Nature Reserve Name & Level & Area & Management agency \\
\hline Hezuo Provincial Forest Park & Provincial & 590.69 & Cooperative City Government \\
\hline Xiahe Ganjia Baishiya Provincial Geopark & Provincial & 5734.2 & Xiahe County Government \\
\hline Yeliguan National Forest Park & National level & 79574.81 & Taohe Ecological Construction Bureau \\
\hline Yeliguan National Geopark & National level & 13840.81 & Lint an County Government \\
\hline Yangsha Provincial Forest Nature Park & Provincial & 5396.03 & Taohe Ecological Construction Bureau \\
\hline Taohe National Nature Reserve, Gansu & National level & 287613.9 & Gansu Forestry and Grass Bureau \\
\hline Jiudongshan Provincial Forest Park, Zhuoni County & Provincial & 748.39 & Zhuoni County Government \\
\hline Gansu Yellow River Shouqu National Nature Reserve & National level & 203057.89 & Gansu Forestry and Grass Bureau \\
\hline $\begin{array}{l}\text { Natural Reserve of Indigenous Fishes in Qinghai-Tibet Plateau, Maqu, } \\
\text { Gansu Province }\end{array}$ & Provincial & 27415.26 & Maqu County Government \\
\hline Gansu Duoer National Nature Reserve & National level & 54401.24 & Gansu Forestry and Grass Bureau \\
\hline Bailongjiang Axia Provincial Nature Reserve, Gansu & Provincial & 130495.88 & Bailong River Forest Management Bureau \\
\hline Zhagana National Geopark, Diebu, Gannan Prefecture & National level & 18357.57 & Diebu County Government \\
\hline Lazikou National Forest Park & National level & 48427.97 & Diebu Ecological Construction Bureau \\
\hline Zhagana Provincial Forest Park, Diebu, Gansu & Provincial & 35896.14 & Diebu County Government \\
\hline Gansu Bailongjiang Chagangliang Provincial Nature Reserve & Provincial & 82915.44 & Bailong River Forest Management Bureau \\
\hline Bailongjiang Boyuhe Provincial Nature Reserve of Gansu & Provincial & 22005.09 & Bailong River Forest Management Bureau \\
\hline Daxiagou National Forest Park & National level & 4110.63 & Zhouqu County Government \\
\hline Laga Mountain Provincial Forest Park & Provincial & 1355.5 & Zhouqu County Government \\
\hline Boyu Provincial Forest Park & Provincial & 13258.81 & Baishui River Ecological Construction Bureau \\
\hline Gansu Gahai-Zecha National Nature Reserve & National level & 247678.63 & Gansu Forestry and Grass Bureau \\
\hline Awancang National Grassland Nature Park, Gansu Province & National level & Unknown & Unknown \\
\hline Gansu Meiren National Grassland Nature Park & National level & Unknown & Unknown \\
\hline
\end{tabular}

\subsection{Related suggestions}

Many problems arose during the process of land consolidation and optimization of natural reserves in Gannan Prefecture, which were resolved through step-by-step negotiation and some common problems were also summarized. Therefore, some suggestions were made for the integration and optimization of natural reserves:

First, it is recommended that the state and the province fully consider the particularities of each region, the complexity of historical issues, and the prominence of actual contradictions. Under the premise of policy permission, it will involve towns, villages, cultivated land, permanent basic farmland and The grassland is transferred out of the protected area to properly resolve historical problems and outstanding contradictions in reality, better consolidate the results of poverty alleviation, promote industrial development, and achieve long-term stability in all regions.

Second, it is recommended to give local governments sufficient decision-making power in the process of integration and optimization, focusing on local governments, further straighten out management systems and mechanisms, clarify management responsibilities and authorities, and better solve the problem of management "two skins" Deal with the relationship between production, life and ecology, and better resolve the actual conflicts between development and protection.

Third, it is recommended that the state and the province further clarify the time limit for withdrawal, and introduce policies and measures to ensure the withdrawal of towns and villages in core protected areas and permanent basic farmland to ensure that the towns and villages in core protected areas and permanent basic farmland withdraw in a planned, orderly, and orderly manner to ensure that farmers Retire, stable, developable, 
rich, and stable.

\section{References}

1. Liu Qi.(2018)Optimized integration and differentiated management and protection Experiences in management of natural protected areas abroad .N. China Natural Resources News, 6

2. Zhang Tongsheng. (2019) The impact of the optimization and integration of nature reserves on scenic spots .J. China Land and Resources Economics, 10: 8-12

3. Tang Fanglin. Wang Mengjun, Sun Hongyan. (2019) The reform path of the management system of nature reserves.J. Forestry Construction, 2:1-5.

4. The General Office of the Central Committee of the Communist Party of China and the General Office of the State Council issued "Guiding Opinions on Establishing a Natural Reserve System with National Parks as the MainBody [EB/OL]. (2019-06-26).[2019-09-03]http://www.gov.cn/zheng ce/2019-06/26/content 5403497.htm.

5. Wang Jing, Mei Yan, Li Jia, Zhao Yanxi, Zhang Yuguang. (2020) The spatial overlap status and integration countermeasures of the Jinfo Mountain Nature Reserve in Chongqing .J.Forestry Inventory and Planning,4: 79-82 